\title{
Características clínicas de los pacientes con injuria renal aguda en diálisis en un hospital general
}

Clinical features of patients with acute kidney injury in dialysis in a general hospital

\author{
Percy Herrera Añazco ${ }^{1,2,3, a}$, Melisa Palacios Guillén ${ }^{4,5, a}$, David Chipayo Gonzales ${ }^{1, b}$, \\ Jorge Gavidia Calderón ${ }^{1, b}$, Manuela Silveira Chau ${ }^{6, c}$ \\ ${ }^{1}$ Hospital Nacional 2 de Mayo, Lima, Perú. \\ ${ }^{2}$ Universidad Científica del Sur, Lima, Perú. \\ ${ }^{3}$ Universidad Nacional de Piura, Piura, Perú. \\ ${ }^{4}$ Hospital Daniel Alcides Carrión, Lima, Perú. \\ ${ }^{5}$ Universidad Nacional Mayor de San Marcos, Lima, Perú. \\ ${ }^{6}$ Clínica Internacional, Lima, Perú. \\ ${ }^{a}$ Médico Nefrólogo; ${ }^{b}$ Residente de Nefrologia; ${ }^{\circ}$ Médico General.
}

\begin{abstract}
Resumen
Introducción: La injuria renal aguda en diálisis es una patología de alta mortalidad en pacientes hospitalizados, especialmente en UCI, y sin estudios actualizados en nuestro medio. Objetivo: Describir las características de los pacientes con insuficiencia renal aguda (IRA) en diálisis de un hospital general. Diseño: Estudio observacional descriptivo unicéntrico. Lugar: Hospital Nacional 2 de Mayo, Lima, Perú. Participantes: Pacientes con IRA en diálisis. Intervenciones: Se estudió a pacientes con IRA en diálisis hospitalizados entre enero y diciembre 2012. Para describir los hallazgos se utilizó promedios, desviaciones estándar y porcentajes. Principales medidas de resultados: Características clínicas de los pacientes con IRA en diálisis. Resultados: Se halló 43 casos de IRA en diálisis, con una edad promedio de 58,5 años (DE: 16,6), de los cuales 28 fueron varones; 20 pacientes (46,5\%) provenían de la UCl. La primera causa de ingreso a diálisis fue oligoanuria $(60,5 \%)$ seguida de encefalopatía urémica $(46,5 \%)$ y acidosis metabólica $(30,2 \%)$. El $39,5 \%$ de todos los pacientes falleció; $45 \%$ de los pacientes que se encontraban en UCI fallecieron. Al alta, 9,3\% de los pacientes continuó en diálisis. Conclusiones: En la población estudiada, 4 de cada 10 pacientes con IRA en diálisis fallecieron y 1 de cada 10 pacientes continuaba en diálisis al alta de hospitalización, en el Hospital Nacional 2 de Mayo.
\end{abstract}

Palabras clave: Injuria renal aguda, diálisis, mortalidad.

\section{Abstract}

Introduction: Acute kidney injury on dialysis has high mortality in hospitalized patients especially in ICU and there are no updated studies in our country. Objectives: To describe characteristics of acute kidney injured patients on dialysis at a general hospital. Design: Descriptive single center observational study. Setting: Hospital Nacional Dos de Mayo, Lima, Peru. Patients: Acute kidney injured (AKI) patients on dialysis. Interventions: We studied patients with AKI on dialysis from January to December, 2012. We described averages, percentages, standard deviations. Main outcome measures: Clinical characteristics of patients with AKI on dialysis. Results: Forty-three acute kidney injured cases on dialysis were reported. Average age was 58.5 years (SD: 16.6), 28 were male, 20 patients (46.5\%) were from ICU. The main cause of dialysis admission was oligoanuria (60.5\%) followed by uremic encephalopathy (46.5\%) and metabolic acidosis (30.2\%); $39.5 \%$ patients died; $45 \%$ of patients in ICU died. At hospital discharge $9.3 \%$ of the patients continued on dialysis. Conclusions: In the population studied 4 of 10 patients with acute kidney injury on dialysis died and 1 of 10 patients continued on dialysis after hospital discharge.

Keywords: Acute kidney injury, dialysis, mortality.

An Fac med. 2013;74(4):307-9

\section{INTRODUCCIÓN}

La injuria renal aguda (IRA) es una patología frecuente en la población hospitalizada ${ }^{(1-3)}$, con alta mortalidad en la población general, pero sobre todo en los pacientes en unidad de cuidados intensivos (UCI) ${ }^{(4,5)}$, de tal forma que en la población general la mortalidad puede llegar a 30 o $40 \%$ y en UCI a 45 o $80 \%{ }^{(4,5)}$, con el consiguiente au- mento de los costos en los sistemas de salud ${ }^{(6)}$.

A pesar de las investigaciones al respecto, no se ha encontrado tratamientos que sean efectivos para el manejo de la IRA, limitándose los esfuerzos a terapias de reemplazo renal (TRR) como la hemodiálisis, cuando es necesaria, y a las recomendaciones para prevenir su aparición ${ }^{(7-9)}$.
En nuestro país no tenemos comunicaciones actualizadas sobre los pacientes con IRA en diálisis, por lo que nos propusimos analizar las características clínicas de los pacientes atendidos con este diagnóstico en el servicio de Nefrología del Hospital Nacional 2 de Mayo (HN2M) durante el año 2012, con el fin de contribuir al conocimiento de la epidemiología actual de esta enfermedad en nuestro país. 


\section{MÉTODOS}

Se realizó un estudio unicéntrico, observacional y prospectivo, entre los meses de enero a diciembre de 2012, de los pacientes que ingresaron a diálisis con diagnóstico de IRA en el servicio de nefrología del HN2M. Se excluyó a los pacientes que ingresaron a diálisis con diagnóstico de enfermedad renal crónica (ERC) o con intoxicación susceptible a diálisis (v.g. intoxicación por metanol).

Se evaluó las siguientes variables de filiación y clínicas, las mismas que fueron registradas en una ficha electrónica: edad, sexo, lugar de procedencia del paciente dentro del hospital (UCI, unidad de cuidados intermedios (UCIN), hospitalización y observación de emergencia), días desde el ingreso al hospital hasta el inicio de diálisis, número de sesiones de diálisis, hemoglobina basal, creatinina, potasio, $\mathrm{pH}$ y bicarbonato. Todos los valores de laboratorio fueron recabados antes del inicio de la diálisis.

Se consignó el diagnóstico de IRA según evaluación del nefrólogo que atendió al paciente y que indicó la diálisis, el mismo que se basó en criterios clínicos como función renal previa y ultrasonografía renal ${ }^{(9)}$. Del mismo modo se consignó la razón de indicación de diálisis según constaba en la historia clínica del servicio de nefrología, definida también por el nefrólogo evaluador.

Utilizando el registro de enfermería del servicio de nefrología del HN2M, se determinó si el paciente había fallecido o si continuó en diálisis al alta.

Se utilizó el software Statistical Package for Social Sciences (SPSS ®) 10,1 para Windows. Para la presentación de resultados en el caso de las variables categóricas se usó frecuencias absolutas y relativas (porcentajes); en el caso de las variables numéricas se determinó medidas de tendencia central y desviación estándar, al evaluarse previamente la normalidad de la distribución.

\section{RESULTADOS}

Desde enero a diciembre de 2012 ingresaron 149 pacientes a diálisis en el servicio de nefrología del HN2M, 43 de los cuales fueron diagnosticados como casos de IRA; todos ingresaron al análisis.

La edad promedio fue 58,5 años (DE: $16,6) ; 28$ de los pacientes fueron varones; 20 (46,5\%) provenían de la UCI, $13(30,2 \%)$ de observación, 7 (16,3\%) de hospitalización y 3 pacientes $(6,9 \%)$ de UCIN.

El tiempo promedio desde su ingreso al hospital y el inicio de diálisis fue 4,4 días (DE: 5,5). Los pacientes tuvieron un número promedio de sesiones de diálisis de 5,04 (DE: 5,69); todos recibieron hemodiálisis convencional.

Los exámenes auxiliares al ingreso son descritos en la tabla 1 y las causas de ingreso a diálisis, en la tabla 2.

Diecisiete pacientes $(39,5 \%)$ fallecieron, 9 en UCI, lo que correspondió a $45 \%$ de los que allí fueron dializados; 5 fallecieron en observación, lo que correspondió a $24,4 \%$ de los allí dializados; 2 en hospitalización $(11,7 \%)$ y 1 en UCIN (5,8\%). Cuatro pacientes $(9,3 \%)$ continuaron en diálisis al alta y una paciente abandonó la terapia durante la hospitalización, por motivos personales.

\section{DISCUSIÓN}

Se ha encontrado un aumento de casos de IRA a nivel mundial, en pacientes hospitalizados, de un promedio de 4,9\% en 1983 hasta 20\% en $2012^{(10,11)}$. Esta prevalencia varía dependiendo de la severidad de la IRA y del lugar donde se está hospitalizado. Así, en UCI se ha hallado una ocurrencia de IRA que requiere TRR entre 5 a $6 \%$, con mortalidad de hasta $80 \%(4,6,12)$.

La mortalidad general encontrada en nuestra población es similar a la de la literatura mundial, la misma que señala mortalidad en IRA severa con o sin TRR entre 20 y $40 \%{ }^{(13,14)}$. Lo mismo
Tabla 1. Valores de laboratorio.

\begin{tabular}{crc} 
Variable & & DE \\
Hemoglobina & $9,68 \mathrm{mg} / \mathrm{dL}$ & 2,553 \\
Creatinina & $5,91 \mathrm{mg} / \mathrm{dL}$ & 3,262 \\
$\mathrm{pH}$ & 7,30 & 0,137 \\
Bicarbonato & $13,38 \mathrm{mEqLL}$ & 4,963 \\
Potasio & $5,67 \mathrm{mEq} / \mathrm{L}$ & 1,590 \\
\hline
\end{tabular}

Tabla 2. Causas de ingreso a diálisis.

\begin{tabular}{ccc} 
Variable & N & $\%$ \\
\hline Oligoanuria & 26 & 60,5 \\
Encefalopatía urémica & 20 & 46,5 \\
Acidosis metabólica & 13 & 30,2 \\
Edema agudo de pulmón & 12 & 27,9 \\
Hiperkalemia & 9 & 20,9 \\
Gastropatía urémica & 3 & 6,9 \\
\hline
\end{tabular}

ocurrió en nuestra población en UCI, donde cerca de la mitad de nuestros pacientes en diálisis falleció.

Si bien es cierto se ha descrito que en países en desarrollo existe un perfil clínico diferente de los pacientes con IRA respecto a los países desarrollados ${ }^{(15,16)}$, también es cierto que esta presentación es bimodal, con diferencias entre los casos en el ámbito rural y en la ciudad ${ }^{(16)}$; eso quizá explique por qué nuestra mortalidad sea igual a la de las comunicaciones en países desarrollados.

No existe un consenso sobre el momento de inicio de la TRR en pacientes con IRA. La reciente guía de la Kidney Disease Improving Global Outcomes (KDIGO) refiere que hay que tomar en cuenta el contexto clínico del paciente y considerar la terapia cuando hay un desbalance en el estado ácido base, de electrolitos y de sobrecarga de fluidos que comprometan la vida ${ }^{(9)}$. La Kidney Disease Outcomes Quality Initiative (KDOQI) reconoce que en esta área aún es necesario estudios bien diseñados para apoyar la decisión de iniciar diálisis ${ }^{\left({ }^{17)}\right.}$. De igual forma, la Sociedad Canadiense de Nefrología señala que, 
en ausencia de una situación que comprometa la vida, el momento del inicio es incierto y un enfoque agresivo del momento de inicio de la TRR podría consumir mayores recursos del sistema de salud ${ }^{(18)}$. Por eso llama la atención que nuestra principal indicación de diálisis sea un criterio que no está relacionado con riesgo de salud directamente. Sin embargo, es posible que el contexto clínico y la coexistencia de otros criterios sea lo que haya primado en la decisión de iniciar la terapia.

La técnica de hemodiálisis también está sujeta, según la KDIGO, a criterios como la inestabilidad hemodinámica del paciente, facilidades logísticas o experiencia del servicio tratante ${ }^{(9)}$. En nuestro hospital, la hemodiálisis convencional fue la técnica usada.

Al igual que en el inicio de la terapia, en el retiro de la misma existen controversias. La KDIGO sugiere que se suspenda la terapia cuando esta ya no sea necesaria, sea porque la función renal intrínseca se ha recuperado hasta el punto de que es adecuada para satisfacer las necesidades del paciente o porque la TRR ya no es consistente con los objetivos de la atención ${ }^{(9)}$. En nuestra población ella fue requerida hasta en un promedio de cinco sesiones de diálisis.

El porcentaje de pacientes que continúa diálisis al alta en nuestra población estuvo acorde con la literatura mundial, donde se refiere que 8 a 22\% de los pacientes críticos que requieren TRR son dados de alta dependiente de diálisis ${ }^{(12,19,20)}$. Situación que ha sido relacionada a la función renal previa, edad, comorbilidades y circunstancias de la IRA ${ }^{(21)}$. La diálisis puede continuar hasta un año después del inicio de la misma hasta en $33 \%$ de los pacientes ${ }^{(22)}$. Sin embargo, esta frecuencia varía con las características propias de la población estudiada. En una revisión sistemática, la incidencia de ERC después de un episodio de IRA fue de 7,8 eventos por paciente por año y la tasa de ERC estadio 5 fue de 4,9 eventos por cada 100 pacientes por año ${ }^{(23)}$.
En nuestro medio se ha publicado experiencias previas sobre casos de IRA, como el de uno de los pioneros de esta especialidad en nuestro país, el doctor Walter Chanamé ${ }^{(24)}$, aunque con criterios de inclusión que difieren con nuestro trabajo, pues la definición y clasificación de la IRA ha cambiado con el tiempo ${ }^{(8,9)}$. Por otro lado, se ha publicado comunicaciones de casos de IRA durante la epidemia del cólera ${ }^{(25-28)}$ e incluso en pacientes postoperados por hipertrofia benigna de próstata ${ }^{(29)}$, sin embargo, de nuestro conocimiento, esta es la primera comunicación de mortalidad general en pacientes de un hospital del Ministerio de Salud (MINSA).

Nuestro estudio es unicéntrico y los hallazgos no pueden ser generalizados a otros centros hospitalarios. De igual forma, al no haber un protocolo de ingreso de pacientes a diálisis en casos de IRA en nuestro hospital, los resultados dependen mucho del criterio del nefrólogo de guardia. Sin embargo, con sus limitaciones, nuestros resultados pueden ser un reflejo de lo que ocurre en los hospitales del MINSA.

En conclusión, 4 de cada 10 pacientes con IRA en diálisis fallecieron y 1 de cada 10 pacientes continuaron en diálisis al alta de hospitalización, en el Hospital Nacional 2 de Mayo.

\section{AGRADECIMIENTOS}

Al servicio de nefrología del Hospital Nacional 2 de Mayo en la persona de su Jefe, el doctor Javier Hernández $\mathrm{Pa}$ checo.

\section{REFERENCIAS BIBLIOGRÁFICAS}

1. Lameire N, Van Biesen W, Vanholder R. The changing epidemiology of acute renal failure. Nat Clin Pract Nephrol. 2006;2(7):364-77.

2. Uchino S, Bellomo R, Goldsmith D, Bates S, Ronco C. An assessment of the RIFLE criteria for acute renal failure in hospitalized patients. Crit Care Med. 2006;34(7):1913-7.

3. Fang $Y$, Ding $X, Z$ Zhong $Y$, Zou J, Teng J, et al. Acute kidney injury in a Chinese hospitalized population. Blood Purif. 2010;30(2):120-6.

4. Clermont G, Acker CG, Angus DC, Sirio CA Pinsky MR, Johnson JP. Renal failure in the ICU comparison of the impact of acute renal failure and endstage renal disease on ICU outcomes. Kidney Int. 2002;62(3):986-96.

5. Liaño F, Pascual J. Outcomes in acute renal failure. Semin Nephrol. 1998;18(5):541-50.

6. Chertow GM, Burdick E, Honour M, Bonventre JB, Bates DW. Acute kidney injury, mortality, length of stay, and costs in hospitalized patients. J Am Soc Nephrol. 2005;16:3365-70.

7. Gaínza FJ, Liaño Garcia F. Guias SEN: Actuación en el fracaso renal agudo. 2007;27(Supl 3):289 pp.

8. UK Renal Association. Clinical practice guidelines module 5: Acute kidney injury. 4th Edition, 2008.

9. Acute Kidney Injury Work Group. Kidney Disease: Improving Global Outcomes (KDIGO). KDIGO Clinical Practice Guideline for Acute Kidney Injury. Kidney Inter. Suppl. 2012; 2:138 pp.

10. Hou SH, Bushinsky D, Wish J B. Hospital-acquired renal insufficiency: a prospective study. Am J Med. 1983;74(2):243-8.

11. Wang HE, Muntner P, Chertow GM, Warnock DG. Acute kidney injury and mortality in hospitalized patients. Am J Nephrol. 2012;35:349-55.

12. Uchino S, Kellum JA, Bellomo R, Doig GS, Morimatsu $\mathrm{H}$, Morguera $\mathrm{S}$, et al. Acute renal failure in critically ill patients: a multinational, multicenter study. JAMA. 2005;294:813-8.

13. Vincent JL, Moreno R, Takala J, Willatts S, De Mendonça A, Bruining $\mathrm{H}$, et al. The SOFA (Sepsisrelated Organ Failure Assessment) score to describe organ dysfunction/failure. On behalf of the working group on sepsis-related problems of the European Society of Intensive Care Medicine. Intensive Care Med. 1996;22:707-10.

14. Murugan R, Kellum J. Acute kidney injury: what's the prognosis? Nat Rev Nephrol. 2011;7(4):209-17.

15. Jha V, Parameswaran S. Community-acquired acute kidney injury in tropical countries. Nat Rev Nephrol. 2013;9(5):278-90.

16. Li PK, Burdmann EA, Mehta RL. Acute kidney injury: global health alert. Am J Kidney Dis. 2013;61(3):359-63.

17. Palevsky PM, Liu KD, Brophy PD, Chawla LS, Parikh CR, Thakar CV, et al. KDOQI US Commentary on the 2012 KDIGO Clinical Practice Guideline for Acute Kidney Injury. Am J Kidney Dis. 2013;61(5):649-72.

18. James M, Bouchard J, Ho J, Klarenbach S, Lafrance JP, Rigatto C, et al. Canadian Society of Nephrology Commentary on the 2012 KDIGO Clinical Practice Guideline for Acute Kidney Injury. Am J Kidney Dis. 2013;61(5):673-85.

19. Bagshaw SM, Laupland KB, Doig CJ, Mortis G, Fick $\mathrm{GH}$, Mucenski M, et al. Prognosis for long-term survival and renal recovery in critically ill patients with severe acute renal failure: a population-based study. Crit Care. 2005;9:700-9.

20. Bell M; SWING, Granath F, Schön S, Ekbom A, Martling CR. Continuous renal replacement therapy is associated with less chronic renal failure than intermittent haemodialysis after acute renal failure. Intensive Care Med. 2007;33:773-80.

21. Liaño F, Felipe C, Tenorio MT, Rivera M, Abraira $\checkmark$, Sáez-de-Urturi JM, et al. Long-term outcome of acute tubular necrosis: a contribution to its natural history. Kidney Int. 2007;71:679-86.

22. Chertow GM, Christiansen CL, Cleary PD, Munro C, Lazarus JM. Prognostic stratification in critically ill patients with acute renal failure requiring dialysis. Arch Intern Med. 1995;155:1505-11 
23. Coca SG, Yusuf B, Shlipak MG, Garg AX, Parikh CR. Long-term risk of mortality and other adverse outcomes after acute kidney injury: a systematic review and meta-analysis. Am J Kidney Dis. 2009;53:961-73.

24. Chanamé $\mathbf{W}$. Tratamiento de la insuficiencia renal aguda con riñón artificial. Hospital Obrero, 1957 a 1972. Tesis Doctoral, Universidad Nacional Mayor de San Marcos (Lima-Perú). 1973.

25. Cieza J, Gutiérrez R, Estremadoyro L, Miyahira J. Insuficiencia renal aguda secundaria a cólera. Aspectos epidemiológicos. Rev Med Hered 1993;3:140-7.
26. Miyahira J, Acosta R, Zurita S, Torres C. Insuficiencia renal aguda en el Hospital Nacional Cayetano Heredia, durante la epidemia de cólera. Rev Med Hered. 1991;2:64-9.

27. Silva H, López F, Alvarado A, Valdez G. Insuficiencia renal aguda durante una epidemia de Cólera, experiencia del Servicio de Nefrologia del Hospital Dos de Mayo. Rev Med Hered. 1991;2(2):70-4.

28. Cieza J, Gamarra G, Torres C. Letalidad y riesgo de insuficiencia renal por cólera en el Hospital Nacional Cayetano Heredia de Lima, Perú. Rev Med Hered. 1991;2(2):75-8.

29. Herrera P, Lozano N, Palacios M, Silveira M. Injuria renal aguda en pacientes pos operados de hipertrofia benigna de próstata. Acta Med Per 2012;29(2):65-9.

Artículo recibido el 18 de mayo de 2013 y aceptado para publicación el 19 de agosto de 2013.

Los autores deben declaran no tener conflictos de interés al presentar este manuscrito.

Correspondencia:

Dr. Percy Herrera Añazco

Olavegoya 1879 dpto 701 Jesús Maria Lima 11 Correo electrónico: silamud@hotmail.com 